\title{
Analysis and Design of Circular Ridged Waveguide Components
}

\author{
Jens Bornemann, Senior Member, IEEE, Smain Amari, Jaroslaw Uher, Member, IEEE, \\ and Rüdiger Vahldieck, Senior Member, IEEE
}

\begin{abstract}
A fast and efficient radial mode-matching technique (RMMT) is applied to the analysis and design of components in circular ridge waveguide technology. Five different structures are investigated with respect to their performance as filters and polarizers. For fast computation, pie-shaped metal ridges and septa are assumed to better fit the cylindrical coordinate system. In practice, the pie-shaped structures are approximated by rectangular cross-section metal inserts. The validity of this approximation is investigated by comparing with measurements and finite-element analysis. It is found that for thin etchable inserts, the measured filter response is in excellent agreement with the theoretical prediction and that for polarizers, the axial ratio response is not particularly sensitive to the ridge shape. Differences between computed and measured results occur only at return loss and isolation levels beyond $25 \mathrm{~dB}$. A central processing unit time comparison with HFSS (4.0) results in a 10-min versus 3-h advantage in favor of the RMMT.
\end{abstract}

Index Terms - Bandpass filters, mode-matching methods, polarization, ridge waveguides.

\section{INTRODUCTION}

C OMPONENTS in circular ridge waveguide (CRW) technology are attractive in the design of front ends for modern satellite and terrestrial communications systems, e.g., [1]-[4], because the circular cross section can be maintained throughout the entire feed system. Since CRW components are comparable in performance with rectangular ridge waveguide components, lossy transitions are avoided and overall system sensitivity is enhanced. In particular, when the metal ridges in CRW are fabricated by etching techniques from thin metal plates and inserted in a split-block housing, the manufacturing process is simplified and higher accuracy can be maintained. Furthermore, the use of ridge sections shortens the guided wavelength, which in turn, reduces the component length considerably.

A major drawback in the past to develop CRW components was the lack of reliable computer-aided design (CAD) procedures. It was difficult to characterize the normally rectangular cross section of the metal ridges within the cylindrical

Manuscript received September 25, 1998; revised November 9, 1998.

J. Bornemann and S. Amari are with the Department of Electrical and Computer Engineering, University of Victoria, Victoria, B.C., Canada V8W 3P6.

J. Uher is with Spar Aerospace Limited, Ste-Anne-de-Bellevue, P.Q., H9X 3R2 Canada.

R. Vahldieck is with the Laboratory for Electromagnetic Fields and Microwave Electronics, Swiss Federal Institute of Technology, CH-8092 Zürich, Switzerland.

Publisher Item Identifier S 0018-9480(99)01950-X. coordinate system. To avoid this mix of coordinate systems, the rectangular ridge cross section was assumed to be radially cut or pie-shaped. Characterizing the mode spectrum of such a structure with various methods has become the focus of several investigations since 1991, e.g., [1], [5]-[9]. Since then, however, only few papers have dealt with the $S$-parameter analysis of transitions from circular waveguide to CRW. An early attempt was published in [2] for bow-tie-shaped metal insert filters (the equivalent to $E$-plane filters in rectangular waveguide) based on a radial mode-matching analysis and, subsequently, in [3] for CRW filters (utilizing the frequencydomain transmission-line matrix (FDTLM) method). In [4], the radial mode-matching technique (RMMT) was extended to calculate $S$-parameters of CRW structures. The work compared the practical realization of the ridges from rectangular metal sheets with the theoretical results assuming ridges with pieshaped cross section. Very good agreement was found, which also confirmed the results in [2].

In this paper, we present a more comprehensive analysis of CRW structures, in which we focus our attention on a filter structure and four different types of polarizers. We are, in particular, interested in investigating the phase-response dependence on the ridge shape. This has not been done before to this extend, but is of great importance in the practical realization of CRW components. Fig. 1 illustrates the following five components under investigation:

1) circular-waveguide metal-insert filter [Fig. 1(a)];

2) septum polarizer [Fig. 1(b)];

3) longitudinal-ridge polarizer [Fig. 1(c)];

4) corrugated-ridge polarizer [Fig. 1(d)];

5) pin polarizer [Fig. 1(e)].

The first four components are fabricated in split-block housing technology, whereas the pin polarizer utilizes pins realized by long screws. (Note that this component has always been experimentally designed so far). Since CRW transformers and evanescent-mode filters have been addressed in [4], that work will not be repeated here.

\section{ANALYSIS AND DESIGN}

The theoretical analysis of the structures in Fig. 1 is based on the RMMT presented in [2] and [4], following the main steps of the mode-matching method, as outlined, e.g., in [10]. Fig. 2 shows the different cross sections involved in the five configurations of Fig. 1. The eigenvalues of the sectoral waveguide [Fig. 2(b) and (c)] are well known [11], and the 


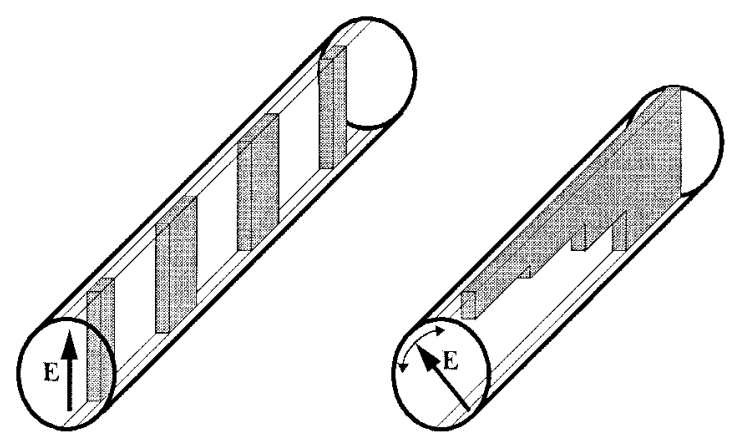

(a)

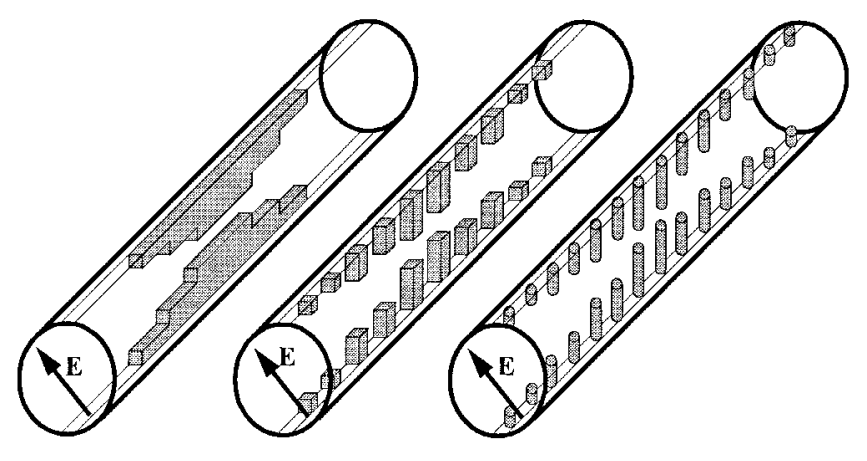

(c)

(d)

(e)

Fig. 1. Ridged circular waveguide components and orientation of the electric field vector at input. (a) Circular waveguide $E$-plane metal-insert filter. (b) Septum polarizer. (c) Longitudinal-ridge polarizer. (d) Corrugated-ridge polarizer. (e) pin polarizer.

eigenvalues of Fig. 2(d)-(f) are determined numerically from a system matrix which is formulated such that a pole-free determinant function is obtained [12]. The field expressions in subregions IIa and b [Fig. 2(d)-(f)], are the same as those in [7]. For the $S$-parameter computation, the reader is referred to [4]. Similar to [13], electric and magnetic walls are placed in the planes of the ridges to accelerate the algorithm. For the components of Fig. 1(c)-(e), this procedure is equivalent to a counterclockwise $45^{\circ}$ rotation [14] of the exciting electric-field vector (cf. Fig. 1).

The design of the CRW components is carried out using a Minimax-based optimization routine (e.g., [15]), except of course for the filter of Fig. 1(a), which lends itself to a quasi-synthesis procedure [10], [16]. Initial values for the circular septum polarizer [Fig. 1(b)] can be found from [1] or by scaling dimensions of similar square polarizers [13] with respect to the fundamental-mode cutoff frequencies of the individual ridged waveguide sections. A comparison to the finite-element-based analysis software package HFSS (4.0) resulted in a 3-h central processing unit (CPU) time job for a three-septum polarizer with 15 frequency points on a SUN Sparc 20. RMMT required $10 \mathrm{~min}$ on a Pentium90 PC for the same task. It should be noted that with increasing frequency points, this ratio will even improve in favor of the RMMT since $75 \%$ of CPU time is tied up with determining the mode spectra of the individual CRW sections.

Since the fast RMMT analysis of CRW sections is based on the assumption that the ridges have a pie-shaped cross section, while in reality the ridge cross section is rectangular, it is

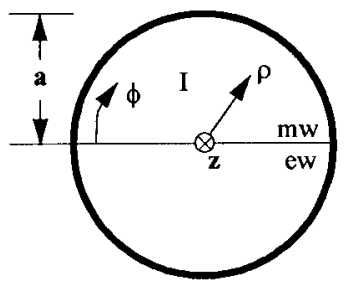

(a)

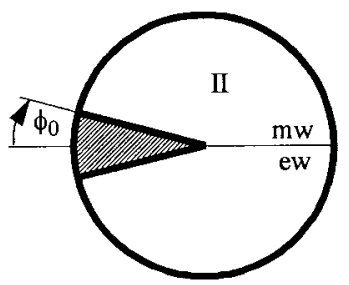

(b)

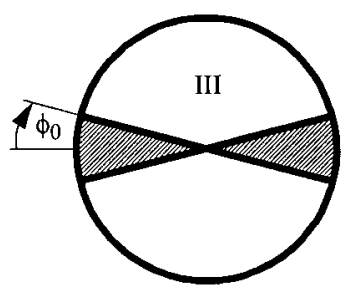

(c)

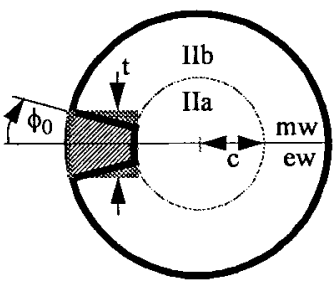

(d)

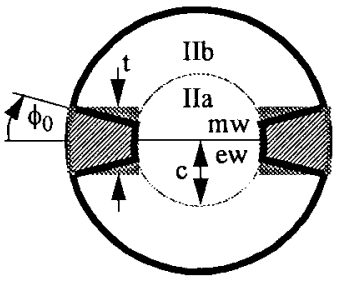

(e)

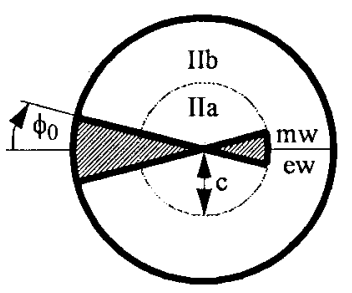

(f)
Fig. 2. Cross sections involved in the mode-matching analysis of ridged circular waveguide components.

important to know up to which ridge thickness this assumption is valid. It should be noted, that although pie-shaped ridges can be fabricated by milling techniques, this is only useful for thick ridge structures. Our objective is to use thin metal plates that can be etched with high accuracy and low cost to the necessary geometry. These plates always have a rectangular shape. The critical point in the analysis that follows is the translation of the nominal ridge thickness $t$ into the semiangle $\phi_{0}$ of the pie-shaped ridge. Our investigations have led to the following conclusions: the components in Fig. 1(a) and (c)-(e) are efficiently calculated by using a constant angle of

$$
\phi_{0}=\arcsin \left(\frac{t}{2 a}\right) \text {. }
$$

The same holds for the septum polarizer of Fig. 1(b) as long as the ridges are fairly thick. For thin and moderately thick ridges in the septum polarizer $(t / a<0.075)$, and only for the cross section of Fig. 2(d), it was found that an approximation that includes the gapwidth leads to slightly better results as follows:

$$
\phi_{0}=\arcsin \left(\frac{t}{2(a+c)}\right) .
$$

Note that, in this case, the semiangle $\phi_{0}$ depends on the penetration depth, thus creating, in theory, additional discontinuities with respect to the ridge thickness. If for any section of the septum polarizer [Fig. 1(b)], subregion IIa [Fig. 2(d) and (f)] becomes extremely small $(c / a<0.02)$, then the computer code replaces that section by the sectoral guide of Fig. 2(b) in order to avoid the possibility of numerical instabilities. 


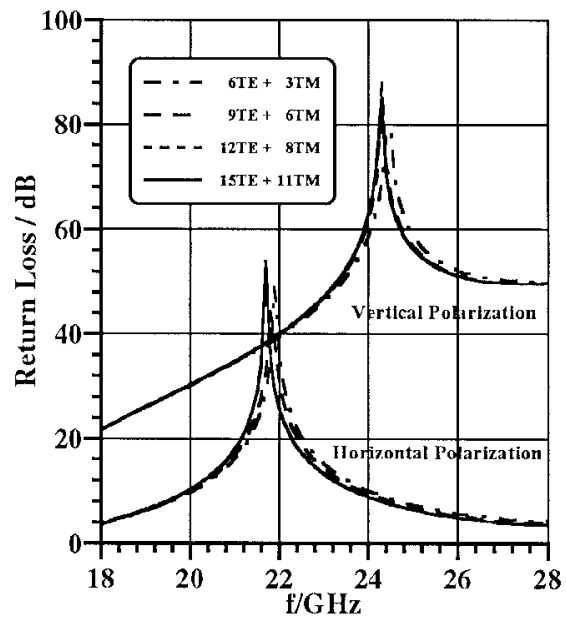

Fig. 3. Input return loss convergence analysis for the structure of Fig. 2(e) between two circular waveguides. Dimensions: Diameter $=0.425^{\prime \prime}$, ridge thickness $=0.016^{\prime \prime}$, gapwidth $=0.2125^{\prime \prime}$, length of ridged section $=0.32^{\prime \prime}$.

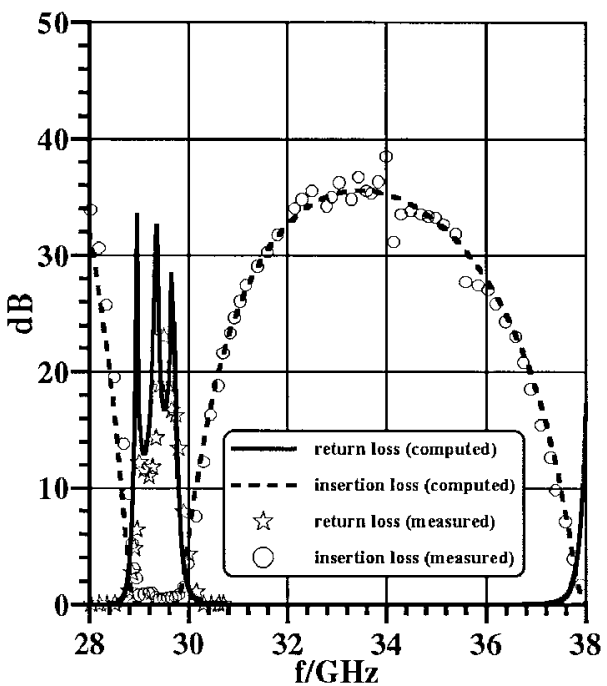

Fig. 4. Measured [16] and computed performance of a three-resonator $K a$-band $E$-plane metal-insert filter according to Fig. 1(a). Dimensions: $a=4.0 \mathrm{~mm}, t=0.15 \mathrm{~mm}$; lengths of inserts: $l_{1}=l_{7}=0.844 \mathrm{~mm}$ $l_{3}=l_{5}=2.985 \mathrm{~mm}$; lengths of resonators: $l_{2}=l_{6}=5.683 \mathrm{~mm}$; $l_{4}=5.768 \mathrm{~mm}$, ridge thickness $t / a=0.0375$.

Fig. 3 shows a return-loss convergence analysis of a doubleridged CRW [Fig. 2(e)] of length 0.32 " axially sandwiched between two circular waveguides. The vertical polarization [perpendicular to the plane of ridges in Fig. 2(e)] is only slightly affected by the ridges. Consequently, the corresponding return loss values are beyond $30 \mathrm{~dB}$ for most of the usable frequency range, and the analysis converges with only 15 symmetric modes $(9 \mathrm{TE}+6 \mathrm{TM})$. The horizontal polarization (parallel to the plane of ridges) is highly affected by the double-ridge insert and, therefore, convergence is reached with 20 symmetric modes $(12 \mathrm{TE}+8 \mathrm{TM})$. Based on this and similar investigations, we have decided to specify the number of TE modes while all TM modes are automatically considered up to the highest TE-mode cutoff frequency. The components in this paper have been calculated by up to 26 modes $(15 \mathrm{TE}+11 \mathrm{TM})$ for the magnetic-wall symmetry and 15 modes $(9 \mathrm{TE}+6 \mathrm{TM})$ for the electric-wall symmetry. Note

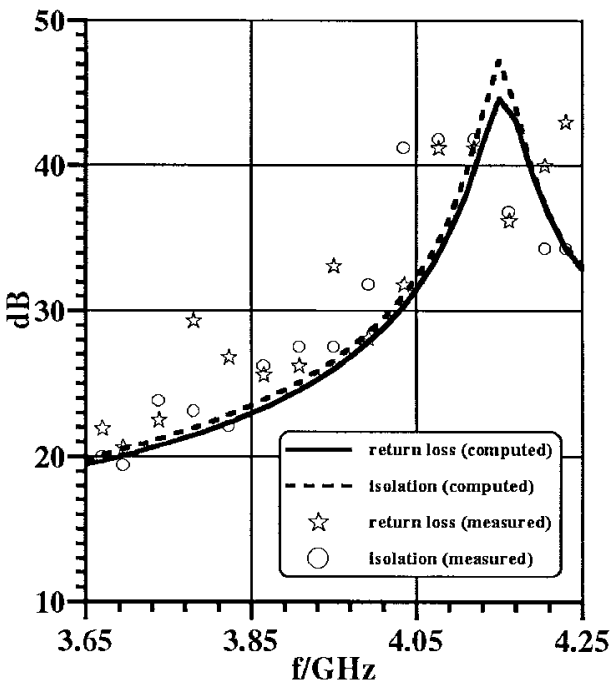

(a)

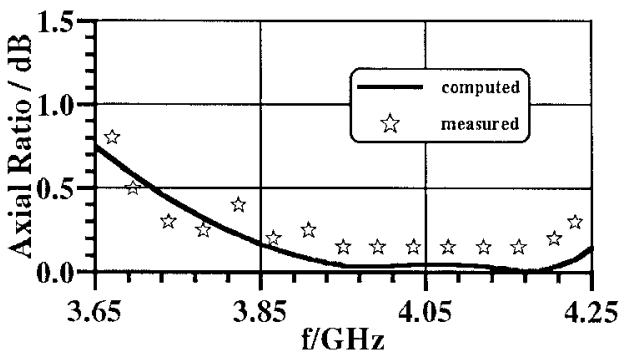

(b)

Fig. 5. Measured and computed performance of a four-section $C$-band septum polarizer [cf. Fig. 1(b)]; ridge thickness $t / a=0.035$. Measurements include transitions to rectangular waveguides at input ports.

that these numbers refer to the respective symmetry planes in the structure; e.g., the septum polarizer of Fig. 1(b) has only one symmetry plane (vertical), whereas the components in Fig. 1(a) and Fig. 1(c)-(e) have an additional horizontal symmetry.

\section{RESULTS}

Several prototypes in CRW technology have been constructed to evaluate the accuracy and reliability of the analysis and design procedure. The first example is a three-resonator $E$ plane metal-insert filter according to Fig. 1(a). Fig. 4 presents the return-loss and insertion-loss measurements [16] in direct comparison with the results of this numerical analysis. Excellent agreement is obtained up to the second passband at about $38 \mathrm{GHz}$. The normalized ridge thickness (thickness-to-radius ratio) is $t / a=0.0375$.

Fig. 5 shows a comparison between measured and calculated results for a four-section $C$-band septum polarizer [Fig. 1(b)] with $t / a=0.035$. The excellent agreement for return loss/isolation (top) and axial ratio (bottom) confirms the validity of the conical-shape approximation. The agreement is the more surprising as the measurements, unlike the theoretical predictions, include the effects caused by transitions from rectangular to quasi-semicircular waveguide at the input ports of the septum polarizer. We attribute this negligible influence to the relatively thin ridge thickness. 


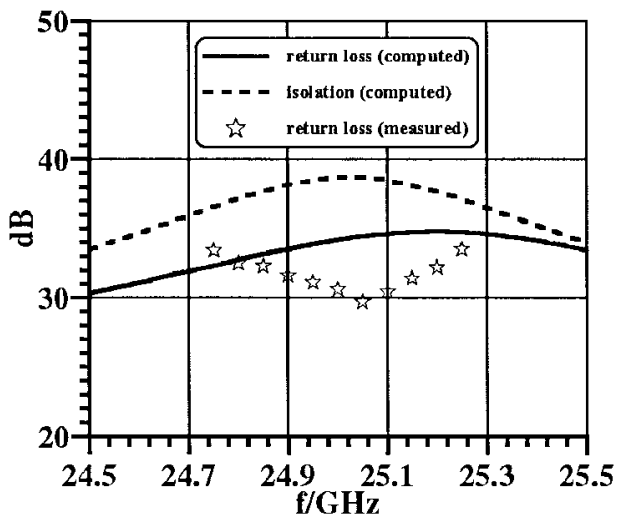

(a)

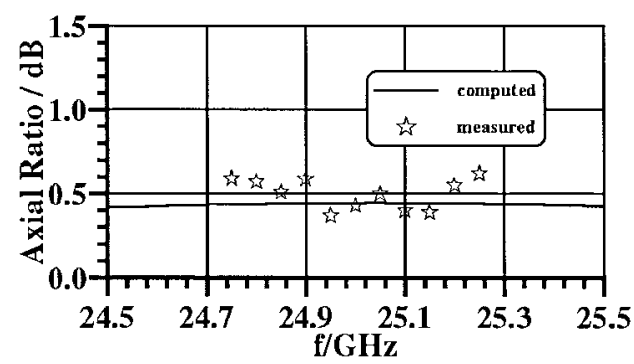

(b)

Fig. 6. Measured and computed performance of a four-section $K$-band septum polarizer [cf. Fig. 1(b)]; ridge thickness $t / a=0.06$. Measurements include transitions to coaxial connectors at input ports.

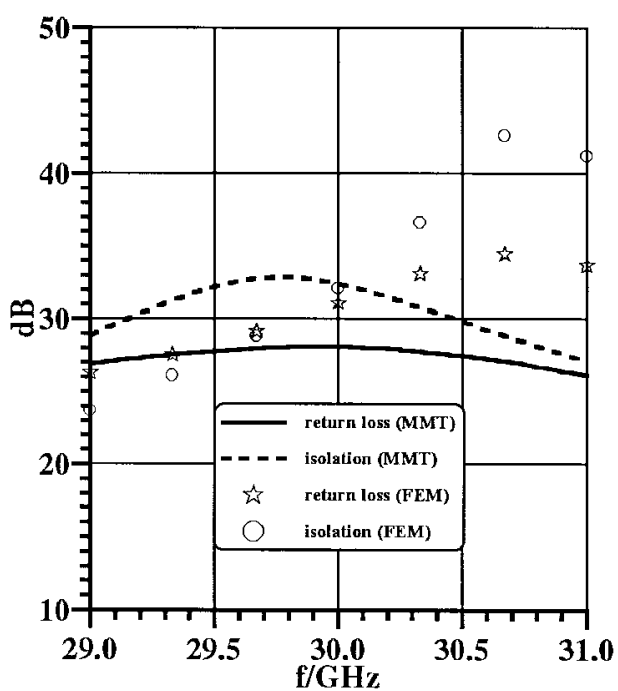

(a)

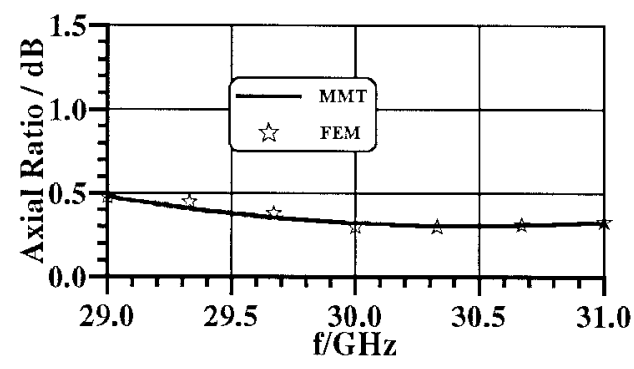

(b)

Fig. 7. Mode-matching and finite-element computations of a four-section $K a$-band septum polarizer [cf. Fig. 1(b)]; ridge thickness $t / a=0.1155$.

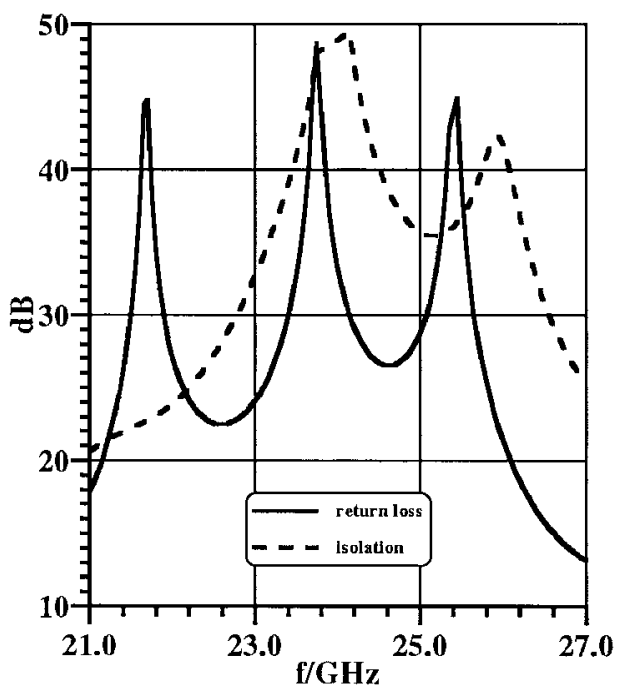

(a)

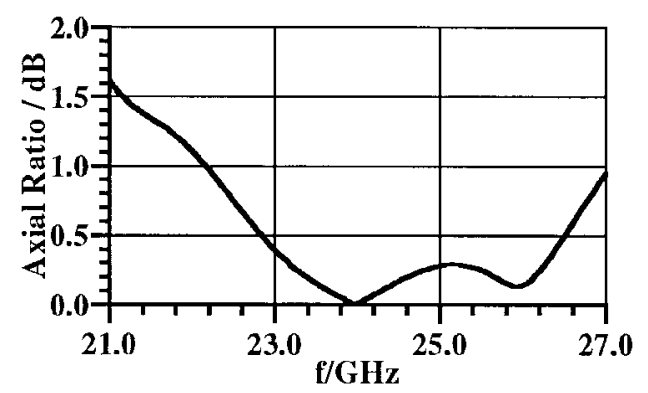

(b)

Fig. 8. Performance of optimized five-section longitudinal-ridge polarizer [cf. Fig. 1(c)]; ridge thickness $t / a=0.07$.

A slightly thicker septum with $t / a=0.06$ has been used in a four-section $25-\mathrm{GHz}$ septum polarizer design. The comparison between measurements and results of the mode-matching design is shown in Fig. 6. Although the general tendency of the return loss curve seems to be reversed, the software correctly predicts more than 30-dB return loss (top). Moreover, the axial ratio computations show very good agreement with the measured values (bottom).

The discrepancies in return loss are believed to be caused by the coax-to-quasi-semicircular waveguide transitions at the input ports of the septum polarizer. These transitions are present in the measurement setup, but are not included in the computations. However, as the ridge thickness increases, the effects of the pie-shape approximation also become more evident. This is demonstrated by a comparison not affected by the measurement setup (Fig. 7). A mode-matching design was carried out for the $30-\mathrm{GHz}$ range and for a ridge thickness of $t / a=0.1155$. Whereas mode matching (conically shaped ridges) usually predicts a smoothly curved return loss (top), the rectangular ridge modeled by the finite-element package HFSS (4.0) is more likely to produce a more pronounced peak. Note that, in spite of these differences, the axial ratio computations [Fig. 7(b)] agree remarkably well.

The performance of a longitudinal-ridge polarizer design is shown in Fig. 8. Design specifications called for 25-dB return loss and 0.3-dB axial ratio between $23.2-25.7 \mathrm{GHz}$. The ridge 


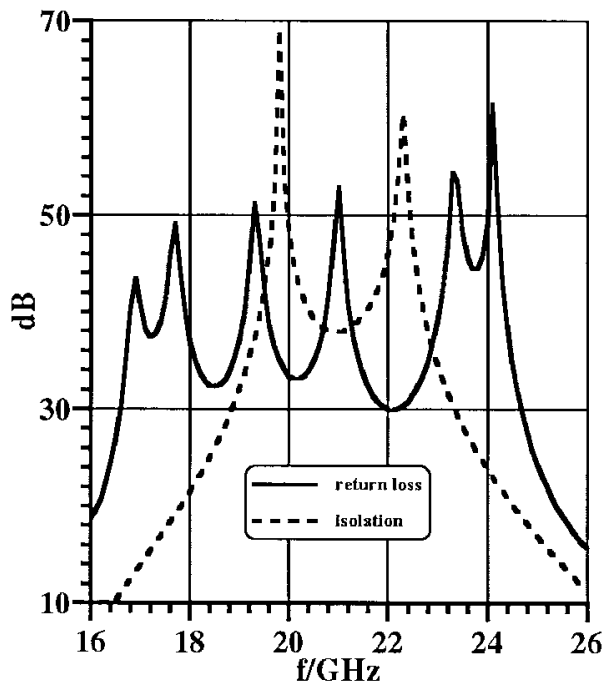

(a)

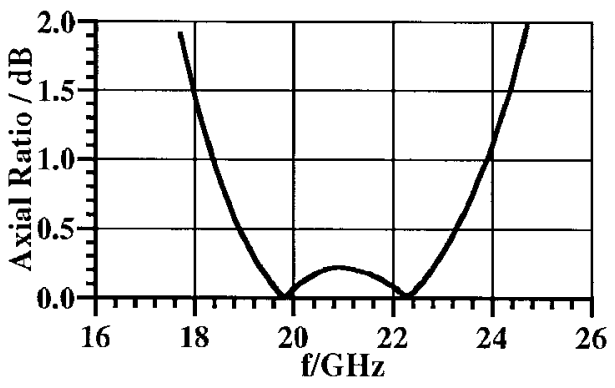

(b)

Fig. 9. Performance of optimized corrugated-ridge polarizer [cf. Fig. 1(d)] with 11 ridges; ridge thickness $t / a=0.07$.

thickness is $t / a=0.07$. Note that the isolation shown here (and in the following figures) is that between the left-handand right-hand-side polarized components at the output of the circular polarizer. It can be determined for "nearly circular polarization" from [10]

$$
X_{\text {pd }}=24.8-20 \log _{10}(\mathrm{ar}) \mathrm{dB}
$$

where ar is the axial ratio in decibels.

Fig. 9 shows the performance of what we refer to as the corrugated-ridge polarizer [cf. Fig. 1(d)]. Eleven ridged sections of thickness $t / a=0.07$ are used with empty circular waveguide sections between them. The optimized $0.3-\mathrm{dB}$ axial ratio bandwidth is $3.7 \mathrm{GHz}$ centered at $21.05 \mathrm{GHz}$. Return loss and isolation are $30 \mathrm{~dB}$ or better over the entire bandwidth.

A pin polarizer prototype [Fig. 1(e)] has been experimentally constructed, and the measurements are shown in Fig. 10 along with the results of the numerical analysis. For the computation, the circular cylindrical pins (screws) are converted to square-cylindrical ones [cf. Fig. 1(d)] with the original diameter taken as the sidelength. Considering the fact that two approximations (circular-to-square pin cross section and rectangular-to-conical ridges) are involved in this case and that the ratio of the pin diameter to the waveguide radius is 0.2 , the agreement is surprisingly good. Again note that the

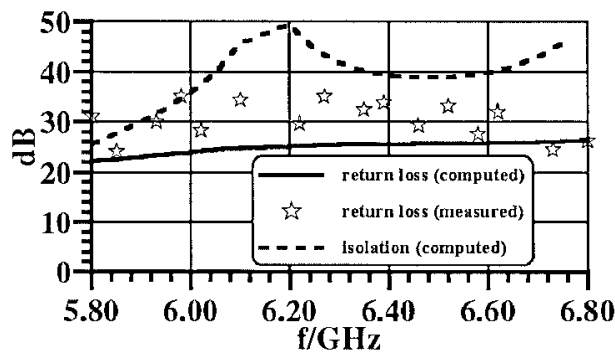

(a)

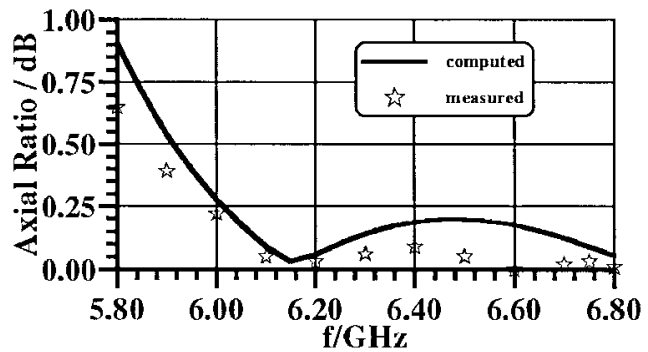

(b)

Fig. 10. Computed and measured performance of a pin polarizer with 11 dual pins [cf. Fig. 1(e)]; pin-diameter $/ a=0.2$. Measurements include transitions to rectangular waveguides at input port.

measurement data include the effects caused by the transition rectangular-to-circular waveguide at the input of the polarizer.

\section{CONCLUSION}

Measurements and comparisons with the finite-element method of different components in ridged circular waveguide technology demonstrate that a mode-matching technique, which approximates rectangular cross-section septa or cylinder-shaped screws by conically shaped ridges, can be used efficiently in design procedures for front-end components. It is found that the axial ratio response is not particularly sensitive to the shape approximation as long as the ridge thickness does not significantly exceed $10 \%$ of the radius of the circular waveguide. Some differences-usually beyond $25 \mathrm{~dB}$ - are observed in the return loss and isolation behavior. Therefore, and in light of the CPU time advantage against commercial field solvers, the mode-matching technique offers a fast and efficient alternative for CRW component design.

\section{REFERENCES}

[1] R. Behe and P. Brachat, "Compact duplexer-polarizer with semicircular waveguide," IEEE Trans. Antennas Propagat., vol. 39, pp. 1222-1224, Aug. 1991.

[2] B. V. de la Filolie and R. Vahldieck, "Coaxial and circular waveguide bandpass filters using printed metal inserts," in IEEE MTT-S Int. Microwave Symp. Dig., 1992, pp. 905-908.

[3] J. Huang, R. Vahldieck, and H. Jin, "Computer-aided design of circular ridged waveguide evanescent-mode bandpass filters using the FDTLM method," in IEEE MTT-S Dig., 1993, pp. 459-462.

[4] U. Balaji and R. Vahldieck, "Mode matching analysis of circular-ridged waveguide discontinuities," IEEE Trans. Microwave Theory Tech., vol. 46, pp. 191-195, Feb. 1998.

[5] M. Guglielmi, R. C. Molina, and A. A. Melcon, "Dual-mode circular waveguide filters without tuning screws," IEEE Microwave Guided Wave Lett., vol. 2, pp. 457-458, Nov. 1992.

[6] W. Schroeder and M. Guglielmi, "Boundary integral equation approach to multi-mode Y-matrix characterization of multi-ridged sections in 
circular waveguide," in IEEE MTT-S Int. Microwave Symp. Dig., 1996, pp. 1849-1852.

[7] U. Balaji and R. Vahldieck, "Radial mode matching analysis of ridged circular waveguides," IEEE Trans. Microwave Theory Tech., vol. 44 pp. 1183-1186, July 1996.

[8] A. Morini, T. Rozzi, and A. Angeloni, "Accurate calculation of the modes of the circular multiridge waveguide," in IEEE MTT-S Int. Microwave Symp. Dig., 1997, pp. 199-202.

[9] H. Z. Zhang and G. L. James, "Cutoff and bandwidth characteristics of a circular waveguide with four symmetrically placed double ridges," in IEEE AP-S Int. Symp. Dig., 1997, pp. 1454-1457.

[10] J. Uher, J. Bornemann, and U. Rosenberg, Waveguide Components for Antenna Feed Systems. Theory and CAD. Norwood, MA: Artech House, 1993.

[11] A. Elsherbeni, D. Kajfez, and S. Zeng, "Circular sectoral waveguides," IEEE Antennas Propagat. Mag., vol. 33, pp. 20-27, Dec. 1991.

[12] S. Amari and J. Bornemann, "A pole-free modal field-matching technique for eigenvalue problems in electromagnetics," IEEE Trans. Microwave Theory Tech., vol. 45, pp. 1649-1653, Sept. 1997.

[13] J. Bornemann and V. A. Labay, "Ridge waveguide polarizer with finite and stepped-thickness septum," IEEE Trans. Microwave Theory Tech., vol. 43, pp. 1782-1787, Aug. 1995.

[14] S. Moretti, F. Alessandri, and R. Sorrentino, "Field theory design of a novel circular waveguide dual-mode filter," in Proc. 25th EuMC, Sept. 1995, pp. 779-783.

[15] K. Madsen, H. Schaer-Jacobsen, and J. Voldby, "Automated minimax design of networks," IEEE Trans. Circuits Syst., vol. CAS-22, pp. 791-796, Oct. 1975 .

[16] B. V. de la Filolie, "Field theory analysis of rectangular and circular waveguide discontinuities for filters, multiplexers and matching networks," Ph.D. dissertation, ECE Dept., Univ. Victoria, Victoria, B.C., Canada, 1992.

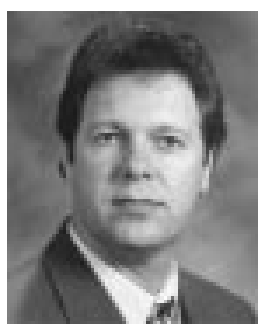

Jens Bornemann (M'87-SM'90) received the Dipl.-Ing. and the Dr.-Ing. degrees in electrical engineering from the University of Bremen, Bremen, Germany, in 1980 and 1984, respectively. From 1984 to 1985, he was a consulting Engineer. In 1985, he joined the University of Bremen, Bremen, Germany, as an Assistant Professor. Since April 1988, he has been with the Department of Electrical and Computer Engineering, University of Victoria, Victoria, B.C., Canada, where he became a Professor in 1992. From 1992 to 1995, he was a Fellow of the British Columbia Advanced Systems Institute. In 1996, he was a Visiting Scientist at Spar Aerospace Limited, Ste-Anne-de-Bellevue, P.Q., Canada, and a Visiting Professor in the Microwave Department, University of Ulm, Ulm, Germany. Since 1997, he has been a Co-Director at the Centre for Advanced Materials and Related Technology (CAMTEC), University of Victoria. He has co-authored Waveguide Components for Antenna Feed Systems: Theory and CAD (Norwood, MA: Artech House, 1993) and has authored and co-authored over 140 technical papers. His research activities include microwave/millimeter-wave components and systems design, and problems involving electromagnetic-field theory in integrated circuits, waveguide feed networks, and radiating structures. He serves on the editorial board of the International Journal of Numerical Modeling.

Dr. Bornemann is a Registered Professional Engineer in the Province of British Columbia, Canada. He is a senior member of IEEE Microwave Theory and Techniques and Antennas and Propagation Societies. He was a recipient of the A. F. Bulgin Premium of the Institution of Electronic and Radio Engineers in 1983. He serves on the editorial board of the IEEE Transactions on MicrowaVe Theory and Techniques.

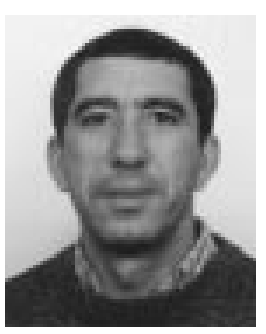

Smain Amari received the D.E.S. degree in physics and electronics from Constantine University, Algeria, in 1985, the M.S. degree in electrical engineering and the Ph.D. degree in physics from Washington University, St. Louis, MO, in 1989 and 1994, respectively.

Since 1994, he has been with the Department of Electrical and Computer Engineering, University of Victoria, Victoria, B.C., Canada. He is interested in numerical methods in electromagnetics, numerical analysis, applied mathematics, applied physics, and application of quantum field theory in quantum many-particle systems.

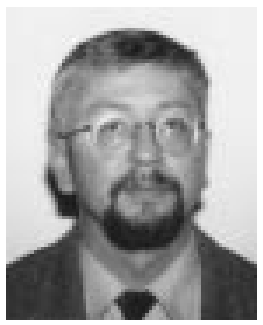

Jaroslaw Uher (M'88) received the Dr.Ing (Ph.D.E.E.) degree in electrical engineering from the University of Bremen, Bremen, Germany, in 1987.

From 1988 to 1990, he was a Post-Doctoral Fellow and Research Engineer at the University of Ottawa, Ottawa, Ont., Canada, where he worked on numerical methods for electromagnetics-modeling of microwave and millimeter-wave components. In 1990, he joined the Antenna Group, Spar Aerospace Ltd., Spar Space Systems, P.Q., Canada, where he is currently a Specialist, Antennna Engineering. His involvement at Spar included various projects such as development of active arrays for satellite communication and remote sensing applications. He co-authored Waveguide Components for Antenna Feed Systems: Theory and CAD (Norwood, MA: Artech House, 1993).

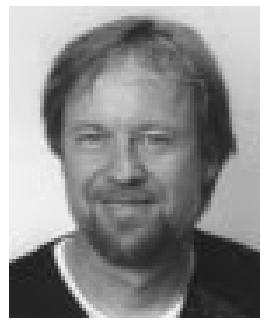

Rüdiger Vahldieck (M'85-SM'86) received the Dipl.-Ing. and the Dr.-Ing. degrees in electrical engineering from the University of Bremen, Bremen, Germany, in 1980 and 1983, respectively.

From 1984 to 1986, he was a Research Associate at the University of Ottawa, Ottawa, Ont., Canada. In 1986, he joined the Department of Electrical and Computer Engineering, University of Victoria, Victoria, B.C., Canada, where he became a Full Professor in 1991. During Fall and Spring 1992-1993, he was a Visiting Scientist at the Ferdinand-BraunInstitute für Hochfrequenztechnik, Berlin, Germany. Since 1997, he has been Chair and Professor for field theory at the Swiss Federal Institute of Technology, Zurich, Switzerland. Since 1981, he has published over 170 technical papers, mainly in the field of microwave CAD. His research interests include numerical methods to model electromagnetic fields for CAD of microwave, millimeter-wave, and opto-electronic integrated circuits. He is also interested in simulation and design of broad-band fiber-optic communication systems and subsystems.

Dr. Vahldieck is the President of the IEEE 2000 International Zurich Seminar on Broad-Band Communications (IZS'2000) and Vice President of the 1999 EMC Zurich. He is a member of the editorial board of the IEEE Transactions on Microwave Theory and Techniques. Since 1992, he has served on the Technical Program Committee of the IEEE International Microwave Symposium and, in 1999, on the TPC of the European Microwave Conference. He is the chairman of the IEEE Swiss Joint Chapter on microwave theory and techniques, antennas and propagation, and electromagnetic compatibility. Together with three coauthors, he received the outstanding publication award of the Institution of Electronic and Radio Engineers in 1983. In 1996, he received the J. K. Mitra Award of the IETE for the best research paper in 1995. 